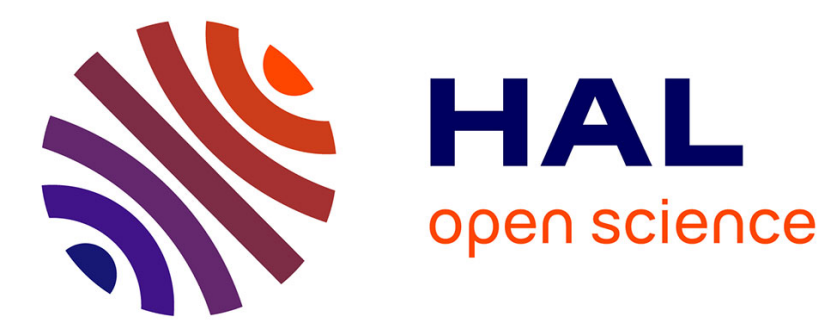

\title{
Dependence of Climate Sensitivity on the Given Distribution of Relative Humidity
}

Stella Bourdin, Lukas Kluft, Bjorn Stevens

\section{To cite this version:}

Stella Bourdin, Lukas Kluft, Bjorn Stevens. Dependence of Climate Sensitivity on the Given Distribution of Relative Humidity. Geophysical Research Letters, 2021, 48 (8), 10.1029/2021GL092462 . hal-03225917

\section{HAL Id: hal-03225917 \\ https://hal.science/hal-03225917}

Submitted on 17 May 2021

HAL is a multi-disciplinary open access archive for the deposit and dissemination of scientific research documents, whether they are published or not. The documents may come from teaching and research institutions in France or abroad, or from public or private research centers.
L'archive ouverte pluridisciplinaire HAL, est destinée au dépôt et à la diffusion de documents scientifiques de niveau recherche, publiés ou non, émanant des établissements d'enseignement et de recherche français ou étrangers, des laboratoires publics ou privés. 


\section{Geophysical Research Letters}

\section{RESEARCH LETTER \\ 10.1029/2021GL092462 \\ Key Points: \\ - Climate sensitivity is sensitive to the assumed distribution of relative humidity (RH) \\ - Different RH profiles explain clear- sky climate sensitivity spread among models \\ - Tropical RH trend in reanalyses yields an increase in climate sensitivity}

Supporting Information:

Supporting Information may be found in the online version of this article.

Correspondence to:

S. Bourdin,

stella.bourdin@lsce.ipsl.fr

Citation:

Bourdin, S., Kluft, L., \& Stevens, B. (2021). Dependence of climate sensitivity on the given distribution of relative humidity. Geophysical Research Letters, 48, e2021GL092462. https://doi. org/10.1029/2021GL092462

Received 18 JAN 2021

Accepted 25 MAR 2021
(C) 2021. The Authors.

This is an open access article under the terms of the Creative Commons Attribution-NonCommercial License, which permits use, distribution and reproduction in any medium, provided the original work is properly cited and is not used for commercial purposes.

\section{Dependence of Climate Sensitivity on the Given Distribution of Relative Humidity}

\author{
Stella Bourdin ${ }^{1,2}$ iD, Lukas Kluft ${ }^{1,3,4}$ iD, and Bjorn Stevens ${ }^{1}$ iD \\ ${ }^{1}$ Max Planck Institute for Meteorology, Hamburg, Germany, ${ }^{2}$ Laboratoire des Sciences du Climat et de l'Environnement, \\ LSCE/IPSL, CEA-CNRS-UVSQ, Université Paris-Saclay, Gif-sur-Yvette, France, ${ }^{3}$ International Max Planck Research \\ School on Earth System Modelling, Hamburg, Germany, ${ }^{4}$ Department of Earth Sciences, Faculty of Mathematics, \\ Informatics and Natural Sciences, Meteorological Institute, Universität Hamburg, Hamburg, Germany
}

Abstract We study how the vertical distribution of relative humidity (RH) affects climate sensitivity, even if it remains unchanged with warming. Using a radiative-convective equilibrium model, we show that the climate sensitivity depends on the shape of a fixed vertical distribution of humidity, tending to be higher for atmospheres with higher humidity. We interpret these effects in terms of the effective emission height of water vapor. Differences in the vertical distribution of RH are shown to explain a large part of the 10\%-30\% differences in clear-sky sensitivity seen in climate and storm-resolving models. The results imply that convective aggregation reduces climate sensitivity, even when the degree of aggregation does not change with warming. Combining our findings with RH trends in reanalysis data shows a tendency toward Earth becoming more sensitive to forcing over time. These trends and their height variation merit further study.

Plain Language Summary Equilibrium Climate Sensitivity is the change in surface temperature in response to a doubling of atmospheric $\mathrm{CO}_{2}$. We study how the assumed vertical distribution of relative humidity affects this sensitivity. Theoretical considerations show that the more moist an atmosphere is, the more it warms as a response to an increase in $\mathrm{CO}_{2}$. Adding water vapor to the lower troposphere has the counter effect, lowering the sensitivity. We emphasize the importance of climate simulations taking humidity into account, as it is largely responsible for the difference in projections among models without clouds. We note surprising trends in humidity-with substantial drying of the lower troposphere over the ocean-in the last four decades as reported by two reanalyses of meteorological observations. Subject to the accuracy of these reconstructions, there appears to be a change with less moistening than expected, but with moistening/drying profiles which will condition Earth to become more sensitive to forcing over time. We stress the need for a study of observations to more critically evaluate these trends, and know better what models should aim for.

\section{Introduction}

The clear-sky response to an increase in greenhouse gases is a pillar of our understanding of global warming (Charney et al., 1979; Manabe \& Wetherald, 1967). It is generally believed that this response is better described by an atmosphere whose relative, rather than absolute, humidity remains constant with warming.

The distinction is crucial because in an atmosphere where the relative humidity (RH) is fixed, the response of surface temperature to radiative forcing (e.g., from changing $\mathrm{CO}_{2}$ ), is roughly twice as large as would be the case should absolute humidity be fixed. In an influential review of these matters, Held and Soden (2000) presented theoretical arguments and evidence from modeling in support of a constant RH. At the time of their review, observations were insufficient to test this hypothesis, but Held and Soden concluded that "10 years may be adequate, and 20 years will very likely be sufficient, [...] to convincingly confirm or refute the predictions." It is now 20 years later.

Taken at face value, two reanalyses of meteorological observations support this point of view, albeit less convincingly than we anticipated. This is shown in Figure 1, where above $600 \mathrm{hPa} \mathrm{RH}$ is increasing with warming, at a rate of $1 \% /$ decade to $4 \%$ /decade. Rather than attempting to establish the reliability of the trends - a task for which we lack expertize-our aim is to estimate their implication for how Earth's equilibrium climate sensitivity may be changing. How does a moister upper, or drier lower, troposphere make 

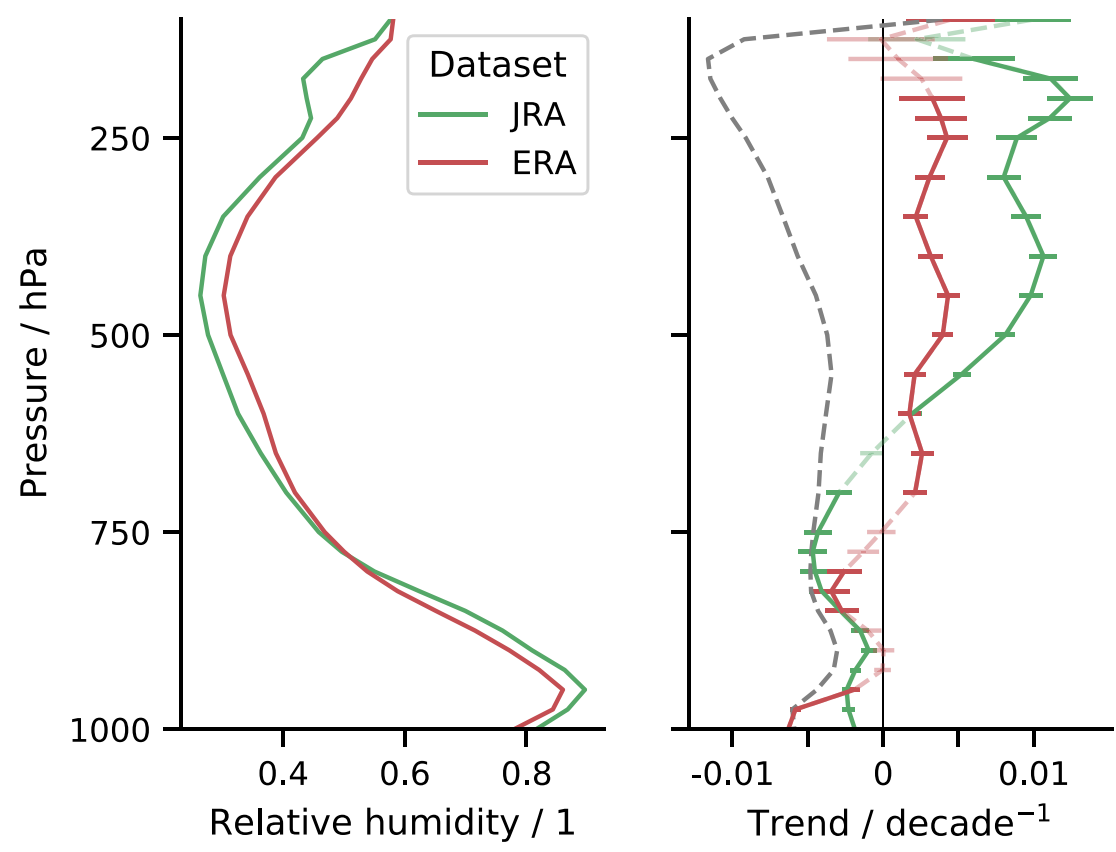

Figure 1. Mean profile (left) and linear trend over 40 years (solid, right) for ERA5 and JRA-55 reanalysis data. Error bars show the 95\% confidence interval for the gradient estimation. Lighter and dashed parts indicate levels for which the null hypothesis of the trend being zero has not been rejected ( $p$-value $>0.01$ ). The gray dashed line corresponds to what would be the trend in RH for a constant absolute humidity considering ERA5 tropical temperature trend. Details about the trend analysis are given in supporting information.

Earth more or less sensitive to forcing? Posing this question raises even more basic questions. For instance, to what extent does the given profile of RH matter for the clear-sky climate sensitivity, even if it remains constant with warming?

If the water vapor pressure, $e$, changes to keep $\mathrm{RH}$ constant with warming, then the fractional vapor pressure change is given by the Clausius-Clapeyron equation, as

$$
\frac{\delta e}{e}=\frac{\ell_{\mathrm{v}}}{R_{\mathrm{v}} T^{2}} \delta T
$$

with $\ell_{\mathrm{v}}$ the vaporization enthalpy, $R_{\mathrm{v}}$ the water vapor gas constant, and $T$ temperature. To the extent the radiative response to an increase in water vapor depends on its fractional change, as for instance is the case for well mixed greenhouse gases (Huang et al., 2016), Equation 1 predicts that this response-and hence the water vapor feedback - should be independent of RH. Support for this point of view is provided by observations and analyses that show outgoing long-wave radiation (OLR) varying linearly with $T$ in a manner that is independent of RH (Koll \& Cronin, 2018; Zhang et al., 2020). These same studies show, however, that the robustness of this relationship is mostly a feature of a colder atmosphere. In the tropics, where continuum emission by water vapor in the 800 to $1,200 \mathrm{~cm}^{-1}$ spectral (window) region becomes more important, $\mathrm{RH}$ begins to color the relationship between OLR and $T$. The tropics cover a substantial portion of the Earth, which raises the question as to whether a sensitivity of the water vapor feedback to the given profile of RH might in part explain differences (15\%, one sigma) in clear-sky feedbacks across climate models (Soden \& Held, 2006; Vial et al., 2013), differences that are even larger across simulations designed to isolate the response of the tropical atmosphere to warming (Becker \& Wing, 2020; Medeiros et al., 2008). Questions such as these have not been the topic of much study. Past work has focused on cloud changes (Sherwood et al., 2020; Stevens et al., 2016), to a degree that can give the impression that clouds alone stand in the way of a meaningful quantification of how surface temperatures, T, respond to radiative forcing. Exceptional is the study by Po-Chedley et al. (2018), 
who argue that changes in $\mathrm{RH}$ in the southern-hemisphere extra-tropics are a large source of model spread; here we emphasize how and why such effects are also substantial in the tropics.

The idea that the climate response is sensitive to the particular distribution of RH being held fixed, can be thought of as a form of state dependence. Perhaps for the reasons given above, most studies addressing this issue adopt a conceptual framework that only admit surface temperature as a state variable (Knutti et al., 2017; Meraner et al., 2013). So long as RH does not vanish, it plays no role.

In the present article we report on our investigation of the influence of $\mathrm{RH}$ on climate sensitivity using a $1 \mathrm{D}$ radiative-convective equilibrium ( $\mathrm{RCE}$ ) model, and highlight a phenomenon we call humidity-dependence. Such a model is attractive for our purposes because it captures (often with surprising fidelity) the behavior of more elaborated descriptions of the climate system in a physically transparent manner. In Section 2 we describe the model and methods. In Section 3 we compute the relative impact of a perturbation in the profile at different levels, as a function of RH. In Section 4 we simulate less idealized profiles of RH to understand and better quantify their effect on the spread in clear-sky climate sensitivity produced by more elaborated models. In Section 5 we return to the trends in the reanalysis RH to quantify their implications for our understanding of the clear-sky climate sensitivity. We conclude in Section 6.

\section{Model and Methods}

Calculations were performed using the 1D-RCE model konrad (Dacie et al., 2019; Kluft et al., 2019). We adopt a configuration that uses the RRTMG radiative scheme (Mlawer et al., 1997) and a hard convective adjustment (Dacie, 2020) following the moist adiabatic lapse rate. Only clear-sky calculations are performed. In a subset of calculations discussed at the beginning of Section 3, we also used a uniform lapse rate. We used 500 pressure levels between 1,000 and $0.5 \mathrm{hPa}$. Following the prescription of the Radiative Convective Equilibrium Model Intercomparison Project(RCEMIP) (Wing et al., 2018), the solar constant is set to $551.58 \mathrm{~W} \mathrm{~m}^{-2}$ and the zenith angle to $42.05^{\circ}$, resulting in an insolation of $409.6 \mathrm{~W} \mathrm{~m}^{-2}$. The surface albedo is 0.2. The ozone profile is defined according to RCEMIP guidelines (Wing et al., 2018), with additional coupling to the cold-point tropopause allowing the ozone layer to shift with troposphere deepening (Kluft, 2020). The RH follows a prescribed vertical distribution up to the cold-point above which the specific humidity is kept uniform at its cold-point value. The $\mathrm{RH}$ is defined with respect to saturation over water above $0^{\circ} \mathrm{C}$ and with respect to saturation over ice below $-23^{\circ} \mathrm{C}$. In between, a combination of both is used (ECMWF, 2018).

A run is defined by its RH profile. It is composed of two equilibrium computations: (i) a spin-up with a constant surface temperature $T_{0}=300 \mathrm{~K}$, (ii) a new equilibrium after applying a sudden doubling of the $\mathrm{CO}_{2}$ concentration. In (ii) the surface has no longer a fixed temperature but a fixed enthalpy sink, whose value is the top of the atmosphere radiative imbalance at the end of the spin-up, as Kluft (2020) argues to be best practice. The equilibrium elimate sensitivity (ECS), $\mathcal{S}$ of our model is defined as the difference between the second equilibrium surface temperature and $T_{0}$.

In Section 3, we discuss perturbation runs. In these, the tropospheric RH profile is uniform except for a $600 \mathrm{~m}$ thick layer, where the $\mathrm{RH}$ is increased or decreased (the perturbation). A perturbation run is thus defined by a base RH, a perturbation pressure, and a perturbation intensity $\delta_{\mathrm{RH}}$. The corresponding "run" without perturbation is called a control run. This is illustrated in Figure 2. Arithmetic changes in RH are adopted, as they correspond to geometric changes in absolute humidity, to which (as a first approximation) the radiative response is proportional, irrespective of the base $\mathrm{RH}$.

As a measure of the impact of a perturbation, we define the amplification factor $a$ as the ratio of the $\mathcal{S}$ in the perturbation run, $\mathcal{S}_{\mathrm{p}}$, to the $\mathcal{S}$ in the corresponding control run, $\mathcal{S}_{\mathrm{c}}$ :

$$
a=\frac{\mathcal{S}_{\mathrm{p}}}{\mathcal{S}_{\mathrm{c}}}-1 .
$$

In reanalysis data, see Figure 1, the RH profiles peak in the boundary layer and in the upper-troposphere and show a distinct minimum in the mid-troposphere. For this reason, we call such a profile $C$-shaped. In 

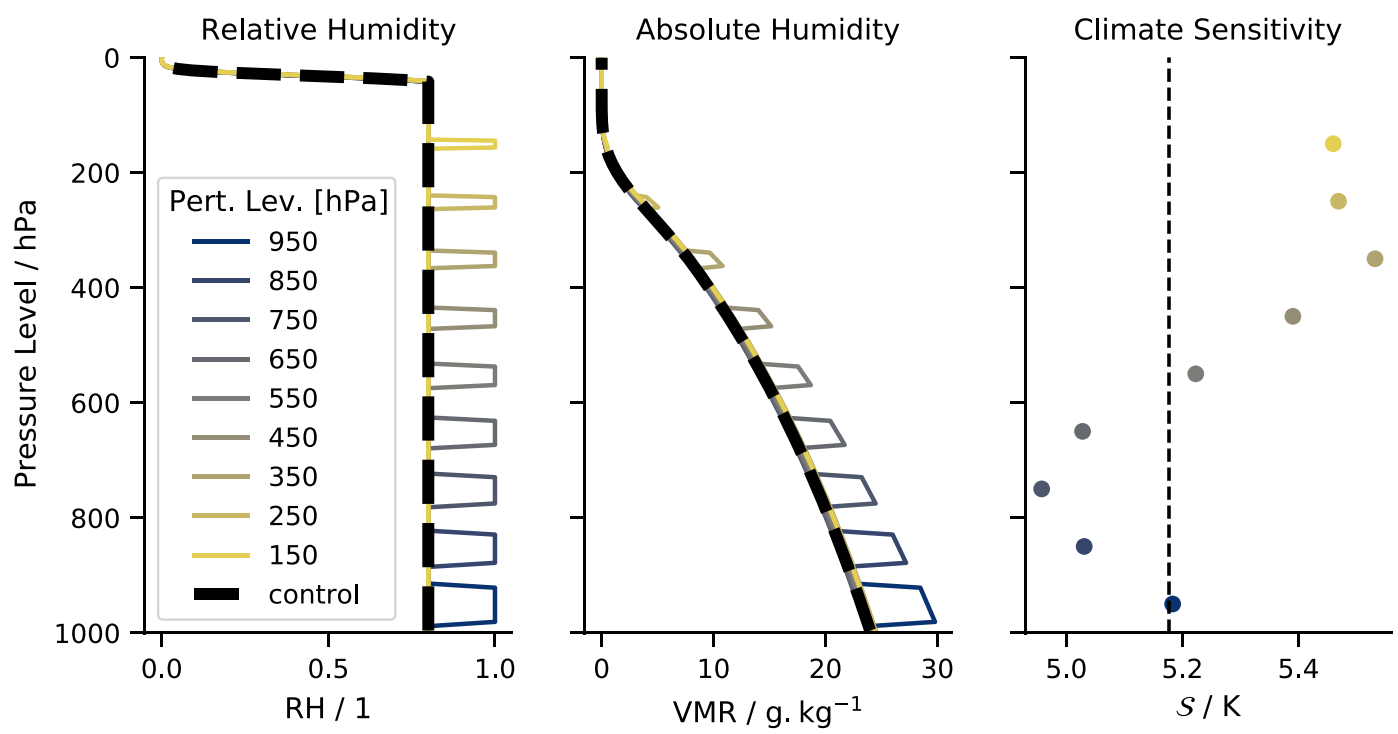

Figure 2. Illustration of the perturbation runs method. The control run, with a base $\mathrm{RH}$ of 0.8 , is shown in dashed black. Each color corresponds to a run with a perturbation $\delta_{R H}=0.2$ at a different level. The two left panels show the relative and absolute humidity profiles. The right panel shows $\mathcal{S}$ for each perturbation run as a function of perturbation pressure alongside the value of $\mathcal{S}$ for the control run (dashed vertical line).

order to simulate a $C$-shaped RH profile, we developed the following piecewise model, in pressure coordinates (shown in Figure 4):

- Linear in the boundary layer, from the surface to the lower-tropospheric peak (low point)

- Quadratic in the mid-troposphere, defined by 3 points: the two peaks and the humidity at $500 \mathrm{hPa}$ (mid point)

- Linear above the upper-tropospheric peak, defined by the upper-tropospheric peak (top point) and the cold-point (which is more strongly coupled to $T$ than $p$ )

The advantages of such an $\mathrm{RH}$ profile is that it is defined by only 5 points, corresponding to parameters that are straightforward to interpret, and it catches the main feature of a realistic profile better than a uniform profile. Moreover, these parameters give us enough degrees of liberty to fit well AMIP and RCEMIP data, as detailed in Section 4.

\section{Humidity-Dependence of $\mathcal{S}$}

To understand how $\mathcal{S}$ varies with mean $\mathrm{RH}$, we first perform runs with different uniform tropospheric $\mathrm{RH}$ profiles, both for the case of uniform and moist adiabatic lapse rates. Values of $\mathcal{S}$ for these runs are plotted in Figure 3 (top panel). We find a robust increase in $\mathcal{S}$ with a moister troposphere. We decomposed $\mathcal{S}$ into contributions from the forcing and the feedback following Gregory et al. (2004). This shows that changes in $\mathcal{S}$ arise from changes in feedback as the forcing tends to be much smaller and of the opposite sign.

Let us use the effective emission height concept for the interpretation of our calculations. Let $\Phi_{\mathrm{e}}$ be Earth's infrared irradiance at the top of the atmosphere. It can be associated with radiant power emitted by a black body at a temperature, $T_{\mathrm{e}}$, such that $\Phi_{\mathrm{e}}=\sigma T_{\mathrm{e}}^{4}$, where $\sigma$ is the Stefan-Boltzmann constant. We define the effective emission height to be the altitude $z_{\mathrm{e}}$ such that $T\left(z_{\mathrm{e}}\right)=T_{\mathrm{e}}$. These ideas can be generalized to allow for spectrally specific effective emission heights, which we denote $z_{e, \lambda}$, as done by Seeley and Jeevanjee (2021).

To help understand the water vapor feedback, we first apply this concept to a case with a uniform lapse rate, $\mathrm{d} T / \mathrm{d} z=-\Gamma$, and gray radiation characterized by a single emission height. If an initial (positive) perturbation in $\mathrm{CO}_{2}$ causes an increase in the emission height $\delta z_{\mathrm{e}, \mathrm{i}}>0$, the troposphere (and surface) warms until $T\left(\delta z_{\mathrm{e}, \mathrm{i}}\right)=T_{\mathrm{e}}$ to maintain $\Phi_{\mathrm{e}}$. As a reaction to this warming, if $\mathrm{RH}$ is to remain fixed, the absolute humidity must increases following Equation 1. The fractional increase in water vapor partial pressure $\delta e / e$ will in 


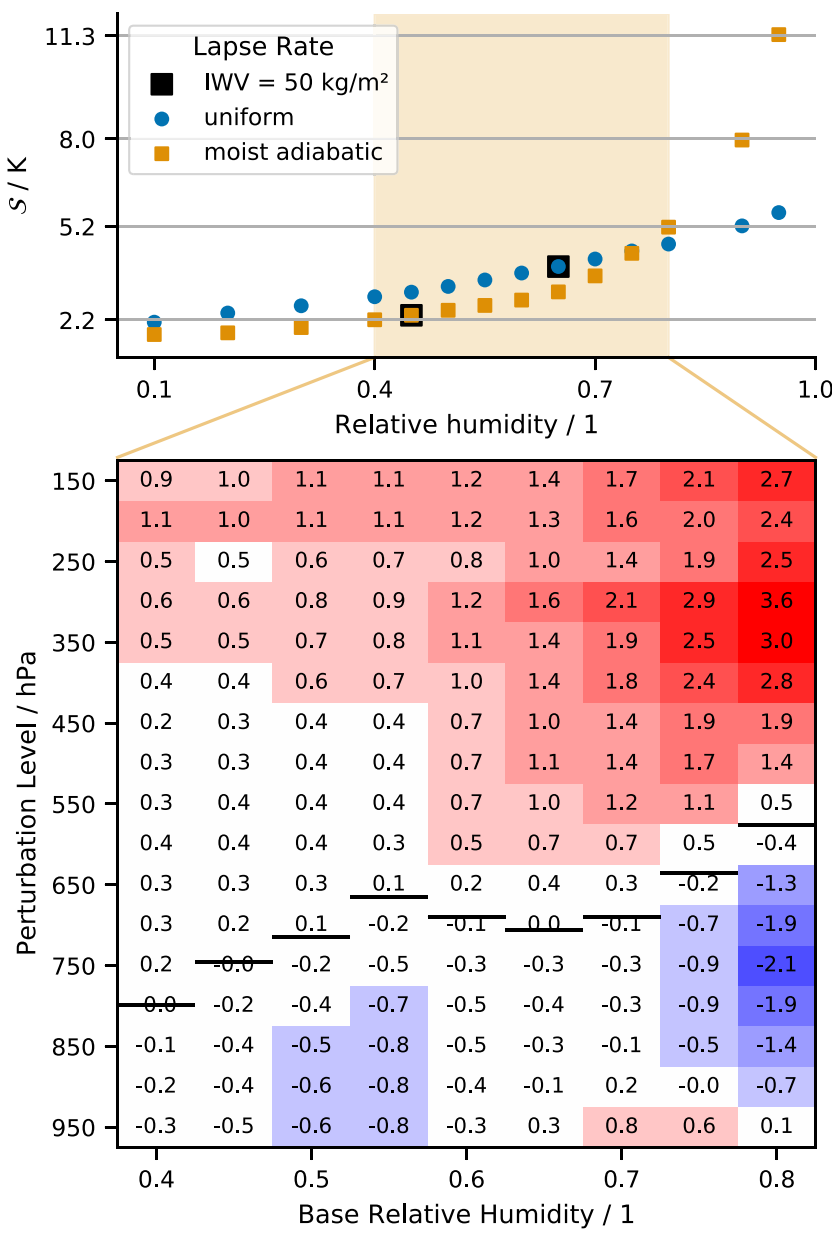

Figure 3. (Upper panel) $\mathcal{S}$ for different uniform tropospheric RH, and for experiments with a uniform tropospheric lapse rate of $6.5 \mathrm{~K} \mathrm{~km}^{-1}$ or with a moist adiabatic lapse rate. Black squared points correspond to experiments were integrated water vapor (IWV) was the closest to $50 \mathrm{~kg} \mathrm{~m}^{-2}$. (Lower panel) Amplification factor $a$ (in percent) for $0.1 \mathrm{RH}$ perturbation for different humidities and different perturbation levels. Blue and red colors for changes larger than $0.5 \%$ in magnitude are indicative of the value's range. Black lines represent the mid-tropospheric level at which $a$ changes sign. turn lead to a further change in $z_{\mathrm{e}}$, which must be balanced by further warming, increasing humidity, and so on. $\mathcal{S}$, is the sum of the response from the initial forcing, plus this water vapor feedback.

The increase in $\mathcal{S}$ with RH (Figure 3) calls into question the line of reasoning (Section 1) leading to the expectation that $\mathcal{S}$ is independent of RH. This may be indicative of continuum emission and absorption by water vapor in the window ( 800 to $1,200 \mathrm{~cm}^{-1}$ ) region, which increases nonlinearly with absolute humidity (Koll \& Cronin, 2018). This would give rise to a stronger humidity-dependence of $\mathcal{S}$ at high temperatures. We thus repeat our calculations at lower values of $T_{0}$, at which the effect of the water vapor continuum is negligible. Reducing the working temperature progressively reduces the sensitivity to $\mathrm{RH}$-at $T_{0}=288 \mathrm{~K} \mathcal{S}$ increases by only $25 \%$ as RH increases from 0.1 to 0.9 -consistent with the hypothesis that the unexpected behavior does indeed stem from the effect of continuum emission in the window region.

The case of the moist adiabat (orange points and matrix in Figure 3) introduces additional complications. A property of the moist adiabat is that the lapse rate monotonically decreases with temperature. This gives rise to three effects: (i) as the atmosphere warms the mean lapse rate becomes smaller (lapse-rate feedback); (ii) for the same surface temperature and mean lapse rate, the moist adiabatic lapse rate is associated with a temperature profile that is everywhere warmer than for the uniform lapse rate; (iii) the lapse-rate is less than the mean in the warmer, lower, troposphere but greater in the colder, upper, troposphere. The first effect gives rise to a negative feedback with warming (smaller $\mathcal{S}$ ) as it implies that the temperature at the emission height warms more rapidly than at the surface. The second effect gives rise to a positive feedback (larger $\mathcal{S}$ ) because a warmer atmosphere will (for the same RH) have a larger absolute humidity. The third effect implies a more bottom heavy humidity profile, which would suggest a slight reduction in $\mathcal{S}$.

At low absolute humidities the negative lapse-rate feedback dominates. This explains why in the upper panel of Figure 3, for $R H \leq 0.75$, runs with a moist-adiabatic lapse rate have a substantially smaller $\mathcal{S}$. This difference becomes even more pronounced (as expected) if one uses the integrated water vapor (IWV) as the control variable. For high humidities the second effect dominates. This occurs because for the chosen value of $T_{0}$ the atmospheric window loses its transparency (Koll \& Cronin, 2018), which is a self-amplifying effect that explains the sharp increase in $\mathcal{S}$. To test our explanation, we performed additional calculations with a smaller $T_{0}$. As the above argument would anticipate, this reduces the sensitivity to $\mathrm{RH}$ (see Figure $\mathrm{S} 3$ in the supporting information), a result that is also consistent with a $\partial_{T_{0}} \mathcal{S}$ decreasing with $T_{0}$, for example, as shown by Meraner et al. (2013). Finally, calculations (not shown) that adopt a constant moist adiabatic lapse rate (which cannot change with warming) also have a slightly reduced $\mathcal{S}$ as compared to calculations adopting a uniform lapse rate for the same value of integrated water vapor (IWV). In this case, there is no lapse-rate feedback and the IWV is the same. This supports our interpretation of the third effect, whereby the shape of the humidity profile also influences $\mathcal{S}$.

To assess how the shape of the RH profile influences $\mathcal{S}$ we perform perturbation runs as described in Section 2 (see also Figure 2). Perturbation runs are performed with $\delta_{\mathrm{RH}}=-0.1,+0.1,+0.2$. From these the amplification factor, $a$ per Equation 2, is related to $\delta_{\mathrm{RH}}$ through linear regression. Figure 3 plots $a$ from its regressed slope multiplied by $\delta_{\mathrm{RH}}=0.1$. Values are calculated for RH perturbations applied every $50 \mathrm{hPa}$ to an otherwise constant $\mathrm{RH}$ profile. This sequence of height varying perturbation runs is computed for $0.4 \leq \mathrm{RH} \leq 0.8$. The impact of a positive $\mathrm{RH}$ perturbation is small, but discernibly positive (increasing $\mathcal{S}$ 

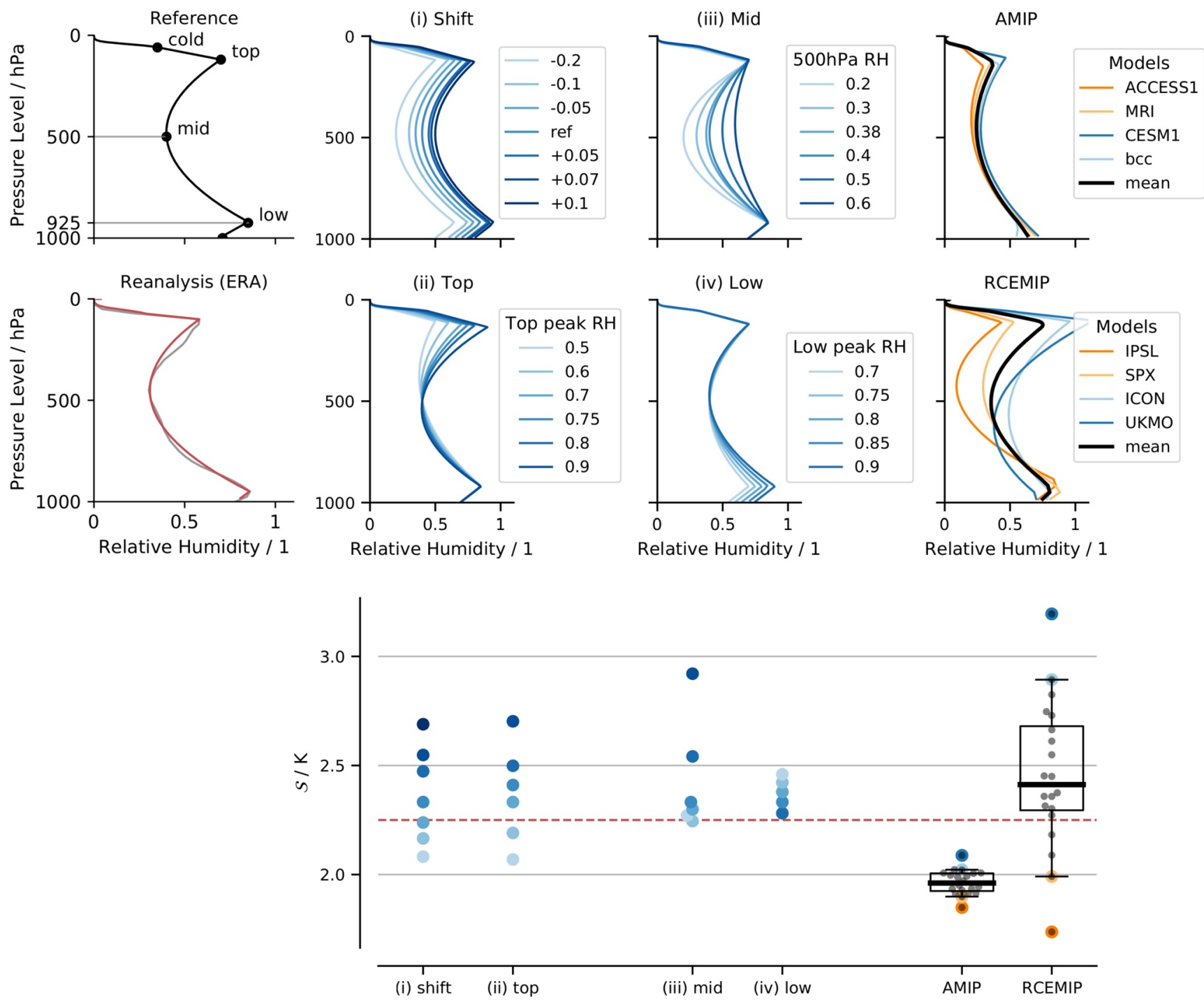

Figure 4. (Upper two rows) $C$-shaped relative humidity $(\mathrm{RH})$ profiles: Reference $0.7 / 0.4 / 0.85$ (top/mid/low) profile (top-left); ERA5 profile as computed for Section 5 (gray), and corresponding $C$-shaped fit (red) (bottom-left). Four central panels correspond to the idealized experiments described in the first paragraph of Section 4. Two right-most panels display the mean and extreme profiles of the AMIP (top-right) and Radiative Convective Equilibrium Model Intercomparison Project (RCEMIP) (bottom-right) data sets. (Lower panel) $\mathcal{S}$ for the idealized experiments and for the experiments with a profile fitted to the AMIP or RCEMIP ensembles. Boxplots' whiskers are set to display the 5th and 95th percentiles. On this graph and for statistics, only one point per model "family" (i.e., issued by the same institute) is used, corresponding to the average of all this family's models. Red dashed line corresponds to the $\mathcal{S}$ computed with ERA5 $C$-shaped fit RH profile above.

) in the upper troposphere, and negative (decreasing $\mathcal{S}$ ) in the lower troposphere. Simulations where the perturbations are fixed relative to the profile of $T$ - which in the mid and upper troposphere is, following Romps (2014), likely to be a more realistic representation of $\mathrm{RH}-$ lead to weaker changes in $\mathcal{S}$, but imply a similar sensitivity to shape. Perturbations at lower values of $p$ (equivalently lower $T$ ) lead to larger increases in $\mathcal{S}$. In every case, the higher the base $\mathrm{RH}$, the stronger is the sensitivity to the humidity perturbation. Moreover, the level of sign change rises with base RH.

The perturbation runs are consistent with our earlier discussion, but not especially intuitive. To understand them, and test their robustness, we performed line-by-line radiative transfer using the ARTS model (Buehler et al., 2018), the results of which are provided graphically in the supporting information (Figure S2). We find two opposing effects. In spectral regions where $z_{e, \lambda}$ is near the height of the RH perturbation, the change in $z_{e, \lambda}$ as water-vapor adjusts to warming is lessened. It is as if the fixed perturbation height helps 
anchor $z_{\mathrm{e}, \lambda}$. In spectral regions where the effective emission height is well below the RH perturbation, the change in $z_{e, \lambda}$ as water-vapor adjusts to warming is heightened-increasing the strength of the water vapor feedback. The first (damping) effect explains the reduction in $\mathcal{S}$ associated with RH perturbations in the lower troposphere. It is also apparent at strongly absorbing wave numbers (rotational and ro-vibrational bands) for the perturbations in the upper troposphere. But for the latter case this reduction is more than offset by the second (amplifying) effect whereby the perturbation in the upper atmosphere increases the changes in $z_{\mathrm{e}, \lambda}$ in parts of the window-region where $\mathrm{CO}_{2}$ does not dominate.

We call humidity-dependence the fact that the climate sensitivity depends on the RH. Not only does the sensitivity depend on the overall humidity, or IWV, but also on the distribution of humidity, that is the shape of the RH profile.

\section{Implications for Model-Based Estimates of ECS}

Given the nonlinearity of these effects, generalization is not automatic. Here, we check whether results of the previous section can also be identified for less idealized perturbations to RH profiles more similar to those observed and simulated by climate models. For this purpose, we use $C$-shaped $\mathrm{RH}$ profiles as defined in Section 2. To reduce their degrees of liberty we additionally fix the low point to $925 \mathrm{hPa}$ and set the slopes below the low point, and above the high point, to $2.0 \times 10^{-5} \mathrm{~Pa}^{-1}$ and $-5.8 \times 10^{-5} \mathrm{~Pa}^{-1}$, respectively. These values are the mean of the parameters when fitting to RCEMIP profiles (see following paragraphs). We additionally set the $\mathrm{RH}$ at the cold-point to be half its peak (upper-troposphere) value, the level of this cold-point being computed by konrad. Calculations (runs) were then performed to quantify the impact of changing the remaining parameters: Starting from a 0.7/0.4/0.85 (top/mid/low) profile, we: (i) shifted the whole profile; (ii) changed only the $\mathrm{RH}$ at the top of the atmosphere; (iii) changed only the humidity at $500 \mathrm{hPa}$; (iv) changed only the humidity in the lower atmosphere. Humidity profiles and resulting changes in $\mathcal{S}$ are presented in Figure 4. Qualitatively the response to these perturbations agrees well with what was learned from the response to more idealized perturbations: (i and ii) $\mathcal{S}$ increases with an increase in the upper tropospheric $\mathrm{RH}$, also when this is part of a general moistening; (iv) $\mathcal{S}$ decreases if $\mathrm{RH}$ increases are confined to the lower troposphere; and (iii) increases in RH in the middle troposphere lead to little change in $\mathcal{S}$ until a critical RH (associated with the closing of the window) is reached, at which point $\mathcal{S}$ begins to increases markedly. The same set of experiments have been performed with a $C$-shaped profile invariant in $T$ in the upper-troposphere, to find very similar results (not shown).

In a second step, we performed runs with RH profiles set to fit RCEMIP simulations using storm-resolving and general circulation models (Except for UKMO-CASIM whose humidity profile led to a runaway) on large domains with an SST of $300 \mathrm{~K}$ (Wing et al., 2020) and CMIP5 AMIP ensembles. The fit is done by retrieving the pressure and humidity of the five points defining our $C$-shaped profile. In particular, the low and top points coincide with the local maxima and the cold-point pressure is retrieved from the temperature profile. The mid point remains fixed at $500 \mathrm{hPa}$ and the surface is taken as the lowest point available. This enables us to assess the effect of the humidity profile alone, all other things being equal.

With RCEMIP RH profiles, we find a $\pm 26 \%$ variation around the mean $\mathcal{S}$ value. The spread in feedback is -1.25 to $-3 \mathrm{~W} \mathrm{~m}^{-2} \mathrm{~K}^{-1}$, slightly smaller but comparable to what is found by Becker and Wing (2020). We thus explain the surprisingly large spread in clear-sky sensitivity in RCEMIP as being in large part a response to different $\mathrm{RH}$ profiles simulated by the models. Both differences in the IWV and the shape of the profiles contribute to the spread, the latter more so, as is also evident by comparing sensitivities calculated using ICON versus the UKMO RH profiles. To the extent that the inter-model spread in RH to different degrees of convective self-aggregation, as claimed by Becker and Wing (2020), our work suggests that different degrees of convective self-aggregation can influence the climate sensitivity, even if the convective self-aggregation does not change with warming.

From CMIP5 AMIP output, we retrieved mean profiles over the tropical oceans (equatorward of $30^{\circ}$ ) averaged over the entire simulated period. As compared to RCEMIP RH profiles, those from the AMIP simulations are on average dryer, and thereby associated with a smaller $\mathcal{S}$. The drier AMIP profiles are indicative of large-scale circulations driven by differences in surface temperatures, i.e., Hadley and Walker cells which 
give rise to the dry tropics. The AMIP simulations differ less in their humidity profiles and likewise show less spread in $\mathcal{S}$, but even so differences approaching $10 \%$ are evident.

Given observations of the RH profiles in the atmosphere, it should be possible to correct model estimates of climate sensitivity using calculations such as ours. From a comparison of Figures 1 and 4, we note that the RCE models tend to be moister than the observations, the AMIP simulations are drier. Fitting the $C$-shaped humidity profile to the observations yields an $\mathcal{S}$ of about $2.25 \mathrm{~K}$; this is smaller than that of most RCE models, but larger than for the AMIP models. Likewise, ECS estimates in early calculations following the RH humidity profile used by Manabe and Wetherald (1967), would, due to an unrealistically dry upper atmosphere, be biased too low. However, for the lower humidities and temperatures used in that study, the fixed lapse assumption actually over compensates, leading to a larger sensitivity as seen in Kluft et al. (2019). This, along with the upper panel of Figure 3, is illustrative of how the lapse rate feedback depends on the base state RH.

\section{Impact of RH Trends in Reanalysis Data}

Based on the above analysis we return to our initial question, which is how to interpret RH trends in the reanalysis products. The profiles presented in Figure 1 are from the ERA5 (Hersbach et al., 2020), and the JRA-55 (Kobayashi et al., 2015) reanalyses of the past 40 years (1979-2019) of meteorological observations. Relative and absolute humidity, as well as temperature, are averaged over tropical oceans (equatorward of $30^{\circ}$ ). Trends regressed from monthly data are significant at several levels and consistent across both reanalyses. They are also evident in the difference between the mean profile in the first and last decade (not shown). We were surprised that RH at low levels was robustly decreasing-something that merits further investigation-even if averaged over height $\delta R H \approx 0$. Our analysis does not tell us how strongly these trends influence the expected warming over the past 40 years, but it does tell us that the pattern of change, with moistening aloft and drying in the lower middle troposphere is conditioning the climate system toward greater sensitivity.

\section{Conclusions}

The response of the atmosphere to radiative forcing as a function of the assumed profile of $\mathrm{RH}$ is explored using a one-dimensional RCE model. For profiles chosen to sample the range produced by state of the art climate and storm-resolving models run under idealized conditions, the calculated equilibrium climate sensitivity of our model $(\mathcal{S})$ varies between $2 \mathrm{~K}$ and $3 \mathrm{~K}$, depending on the $\mathrm{RH}$ profile, highlighting a humiditydependence of the climate sensitivity: Moister atmospheres were shown to have a larger $\mathcal{S}$, increasingly so with warmer temperature, consistent with understanding of how water vapor influences the transmissivity of the atmospheric window (Koll \& Cronin, 2018; Nakajima et al., 1992; Seeley \& Jeevanjee, 2021). $\mathcal{S}$ is further shown to increase with increasing humidity in the upper troposphere, but decreases with increases in humidity in the lower mid-troposphere.

The use of a simple physical model, konrad, makes it easier to understand the basic physics determining the outcome of our calculations. For instance, with the chosen framework it is possible to show how the lapse rate's influence on the total amount and vertical distribution of humidity for a given profile of RH influences $\mathcal{S}$. We could also investigate how $\mathcal{S}$ depends on the shape of the $\mathrm{RH}$ profile, which expresses competing effects, whereby perturbations to the humidity can both reduce or increase the change in the emission height associated with changes in absolute humidity to maintain a constant RH with warming. The former effect dominates when the humidity perturbation is near the emission height resulting in a slight reduction in $\mathcal{S}$ for bottom heavy humidity profiles.

Our work emphasizes the importance of realistically representing the $\mathrm{RH}$ profile when calculating climate sensitivity. Models that are too humid, particularly in the mid- and upper-troposphere will have larger sensitivities, an effect which will amplify with increased warming. Convective self-aggregation modifies the mean RH profile, thereby reducing ECS, even if the degree of convective aggregation itself does not change with warming. In this context, our study also encourages the use of RH as metric of the fidelity of the moist 
physics in climate models. To the extent climate models are unable to realistically represent the observed distribution of RH, our methods may make it possible to estimate the quantitative effect of these biases.

Humidity profiles over tropical oceans as represented in reanalysis products, tend to be moister than those produced by models forced with observed SSTs, implying a larger clear-sky sensitivity. Three dimensional radiative convective equilibrium models, which are more physical-but less constrained by large-scale sea-surface temperature gradients-tend to be more humid, but also have more divergent humidity profiles.

Surprisingly large changes in $\mathrm{RH}$ are reported by the reanalysis products over the last 40 years, changes which our calculations suggest will condition the climate system to be more sensitive to forcing in the future. This finding adds an additional dimension to Knutti and Rugenstein's (2015) statement that the feedback parameter is not constant, and that nonlinearity in the system may be important when assessing Earth's equilibrium climate sensitivity. The surprising trends in the reanalysis humidity products, particularly the drying in the tropical lower troposphere, reminds us of Held and Soden's plea to be attentive to this issue, and merits the renewed attention of experts.

\section{Conflict of Interest}

The authors declare no conflict of interest.

\section{Data Availability Statement}

Primary data including simulation scripts and code for reproducing the figures are available on Zenodo through https://doi.org/10.5281/zenodo.4559581; konradv0.8.1 is available at github.com/atmtools/konrad, and latest sources are available at https://doi.org/10.5281/zenodo.4434837; ERA5 data is available on the Copernicus Climate Change Service Climate Data Store (CDS, https://cds.climate.copernicus.eu/cdsapp\#!/ dataset/reanalysis-era5-pressure-levels-monthly-means?tab=overview); JRA-55 data were retrieve from https://jra.kishou.go.jp/JRA-55/index_en.html; The German Climate Computing Center (DKRZ) hosts the standardized RCEMIP and CMIP5-AMIP output (https://cera-www.dkrz.de/WDCC/ui/cerasearch/ info?site=RCEMIP_DS).

\section{References}

The research is supported by public funding to the Max Planck Society. S. Bourdin also benefited from the French state aid managed by the ANR under the "Investissements d'avenir" programme with the reference ANR-11IDEX-0004-17-EURE-0006. B. Stevens and L. Kluft acknowledge support from the European Union's Horizon 2020 Research and Innovation Programme under grant agreement No. 820829 (CONSTRAIN Project). The authors thank Tobias Becker, Sally Dacie, Didier Paillard, Maria Rugenstein, and Catherine Stauffer for helpful scholar discussion concerning this study, and Hauke Schmidt's GCC group at MPI-M for advice and feedback. The authors are also grateful to Sébastien Fromang and Theresa Lang for their review of an earlier version of this manuscript, as well as the stimulating and thoughtful comments of Nadir Jeevanjee and an anonymous reviewer, which led to a deeper understanding of our results.
Becker, T., \& Wing, A. A. (2020). Understanding the extreme spread in climate sensitivity within the radiative-convective equilibrium model intercomparison project. Journal of Advances in Modeling Earth Systems, 12(10), e2020MS002165. https://doi. org/10.1029/2020MS002165

Buehler, S. A., Mendrok, J., Eriksson, P., Perrin, A., Larsson, R., \& Lemke, O. (2018). ARTS, the atmospheric radiative transfer simulatorversion 2.2, the planetary toolbox edition. Geoscientific Model Development, 11(4), 1537-1556. https://doi.org/10.5194/gmd-11-1537-2018

Charney, J. G., Arakawa, A., Baker, D. J., Bolin, B., Dickinson, R. E., Goody, R. M., et al. (1979). Carbon dioxide and climate: A scientific assessment. National Academy of Sciences.

Dacie, S. (2020). Using simple models to understand changes in the tropical mean atmosphere under warming. University of Hamburg. (Unpublished doctoral dissertation).

Dacie, S., Kluft, L., Schmidt, H., Stevens, B., Buehler, S. A., Nowack, P. J., et al. (2019). A 1D RCE study of factors affecting the tropical tropopause layer and surface climate. Journal of Climate, 32(20), 6769-6782. https://doi.org/10.1175/JCLI-D-18-0778.1

ECMWF. (2018). Part IV: Physical processes. In IFS documentation CY45R1. ECMWF. Retrieved from https://www.ecmwf.int/node/18714

Gregory, J. M., Ingram, W. J., Palmer, M. A., Jones, G. S., Stott, P. A., Thorpe, R. B., et al. (2004). A new method for diagnosing radiative forcing and climate sensitivity. Geophysical Research Letters, 31(3), L03205. https://doi.org/10.1029/2003GL018747

Held, I. M., \& Soden, B. J. (2000). Water vapor feedback and global warming. Annual Review of Energy and the Environment, 25(1), 441. https://doi.org/10.1146/annurev.energy.25.1.441

Hersbach, H., Bell, B., Berrisford, P., Hirahara, S., Horányi, A., Muñoz-Sabater, J., et al. (2020). The ERA5 global reanalysis. Quarterly Journal of the Royal Meteorological Society, 146(730), 1999-2049. https://doi.org/10.1002/qj.3803

Huang, Y., Tan, X., \& Xia, Y. (2016). Inhomogeneous radiative forcing of homogeneous greenhouse gases. Journal of Geophysical Research: Atmospheres, 121(6), 2780-2789. https://doi.org/10.1002/2015JD024569

Kluft, L. (2020). Benchmark calculations of the climate sensitivity of radiative-convective equilibrium. Doctoral dissertation Universität Hamburg. https://doi.org/10.17617/2.3274272

Kluft, L., Dacie, S., Buehler, S. A., Schmidt, H., \& Stevens, B. (2019). Re-examining the first climate models: Climate sensitivity of a modern radiative-convective equilibrium model. Journal of Climate, 32, 8111-8125. https://doi.org/10.1175/JCLI-D-18-0774.1

Knutti, R., \& Rugenstein, M. A. A. (2015). Feedbacks, climate sensitivity and the limits of linear models. Philosophical Transactions of the Royal Society A Mathematical, Physical and Engineering Sciences, 373(2054), 20150146. https://doi.org/10.1098/rsta.2015.0146

Knutti, R., Rugenstein, M. A. A., \& Hegerl, G. C. (2017). Beyond equilibrium climate sensitivity. Nature Geoscience, 10(10), 727-736. https://doi.org/10.1038/ngeo3017 
Kobayashi, S., Ota, Y., Harada, Y., Ebita, A., Moriya, M., Onoda, H., et al. (2015). The JRA-55 reanalysis: General specifications and basic characteristics. Journal of the Meteorological Society of Japan, 93(1), 5-48. https://doi.org/10.2151/jmsj.2015-001

Koll, D. D. B., \& Cronin, T. W. (2018). Earth's outgoing longwave radiation linear due to $\mathrm{H}_{2} \mathrm{O}$ greenhouse effect. Proceedings of the National Academy of Sciences of the United States of America, 115(41), 10293-10298. https://doi.org/10.1073/pnas.1809868115

Manabe, S., \& Wetherald, R. T. (1967). Thermal Equilibrium of the atmosphere with a given distribution of relative humidity. Journal of the Atmospheric Sciences, 24, 241-259. https://doi.org/10.1175/1520-0469(1967)024〈0241:TEOTAW $\rangle 2.0 . C O ; 2$

Medeiros, B., Stevens, B., Held, I. M., Zhao, M., Williamson, D. L., Olson, J. G., \& Bretherton, C. S. (2008). Aqua planets, climate sensitivity, and low clouds. Journal of Climate, 21(19), 4974-4991. https://doi.org/10.1175/2008JCLI1995.1

Meraner, K., Mauritsen, T., \& Voigt, A. (2013). Robust increase in equilibrium climate sensitivity under global warming. Geophysical Research Letters, 40(22), 5944-5948. https://doi.org/10.1002/2013GL058118

Mlawer, E. J., Taubman, S. J., Brown, P. D., Iacono, M. J., \& Clough, S. A. (1997). Radiative transfer for inhomogeneous atmospheres: RRTM, a validated correlated-k model for the longwave. Journal of Geophysical Research, 102(D14), 16663-16682. https://doi. org/10.1029/97JD00237

Nakajima, S., Hayashi, Y.-Y., \& Abe, Y. (1992). A study on the "runaway greenhouse effect" with a one-dimensional radiative-convective equilibrium model. Journal of the Atmospheric Sciences, 49(23), 2256-2266. https://doi.org/10.1175/1520-0469(1992)049/2256:A SOTGE $>2.0 . \mathrm{CO} ; 2$

Po-Chedley, S., Armour, K. C., Bitz, C. M., Zelinka, M. D., Santer, B. D., \& Fu, Q. (2018). Sources of intermodel spread in the lapse rate and water vapor feedbacks. Journal of Climate, 31(8), 3187-3206. https://doi.org/10.1175/JCLI-D-17-0674.1

Romps, D. M. (2014). An analytical model for tropical relative humidity. Journal of Climate, 27(19), 7432-7449. https://doi.org/10.1175/ jcli-d-14-00255.1

Seeley, J. T., \& Jeevanjee, N. (2021). $\mathrm{H}_{2} \mathrm{O}$ windows and $\mathrm{CO}_{2}$ radiator fins: A clear-sky explanation for the peak in equilibrium climate sensitivity. Geophysical Research Letters, 48, e2020GL089609. https://doi.org/10.1029/2020GL089609

Sherwood, S. C., Webb, M. J., Annan, J. D., Armour, K. C., Forster, P. M., Hargreaves, J. C., et al. (2020). An assessment of Earth's climate sensitivity using multiple lines of evidence. Reviews of Geophysics, 58, e2019RG000678. https://doi.org/10.1029/2019RG000678

Soden, B. J., \& Held, I. M. (2006). An assessment of climate feedbacks in coupled ocean-atmosphere models. Journal of Climate, 19(14), 3354-3360. https://doi.org/10.1175/JCLI3799.1

Stevens, B., Sherwood, S. C., Bony, S., \& Webb, M. J. (2016). Prospects for narrowing bounds on Earth's equilibrium climate sensitivity. Earth's Future, 4(11), 512-522. https://doi.org/10.1002/2016EF000376

Vial, J., Dufresne, J.-L., \& Bony, S. (2013). On the interpretation of inter-model spread in CMIP5 climate sensitivity estimates. Climate Dynamics, 41(11), 3339-3362. https://doi.org/10.1007/s00382-013-1725-9

Wing, A. A., Reed, K. A., Satoh, M., Stevens, B., Bony, S., \& Ohno, T. (2018). Radiative-convective equilibrium model intercomparison project. Geoscientific Model Development, 11, 793-813. https://doi.org/10.5194/gmd-11-793-2018

Wing, A. A., Stauffer, C. L., Becker, T., Reed, K. A., Ahn, M.-S., Arnold, N. P., et al. (2020). Clouds and convective self-aggregation in a multimodel ensemble of radiative-convective equilibrium simulations. Journal of Advances in Modeling Earth Systems, 12(9), e2020MS002138. https://doi.org/10.1029/2020ms002138

Zhang, Y., Jeevanjee, N., \& Fueglistaler, S. (2020). Linearity of outgoing longwave radiation: From an atmospheric column to global climate models. Geophysical Research Letters, 47(17), e2020GL089235. https://doi.org/10.1029/2020GL089235 